\title{
Latitudinal Temperature Variations of Jovian $\mathrm{H}_{3}^{+}$
}

\author{
Gilda E. Ballester \\ Department of Atmospheric, Oceanic and Space Sciences, University of Michigan, Ann Arbor, Michigan 48109-2143; and Department of \\ Physics and Astronomy, University College London, Gower Street, London WCIE 6BT, United Kingdom \\ Steven Miller and Jonathan Tennyson \\ Department of Physics and Astronomy, University College London, Gower Street, London WCIE 6BT, United Kingdom \\ E-mail: sm7@ukacrl \\ Laurence M. Trafton \\ McDonald Observatory, Astronomy Department, University of Texas at Austin, Austin, Texas 78712
}

AND

Thomas R. Geballe

Joint Astronomy Centre, 660 N. A'ohoku Place, University Park, Hilo, Hawaii 96720

Received May 17, 1993; revised November 10, 1993

\begin{abstract}
A spectral image of Jupiter centred on $3.45 \mu \mathrm{m}$, taken on the night of April 2, 1992, using cooled grating spectrometer CGS4 on the United Kingdom Infrared Telescope, shows that features due to the $\nu_{2}$ ro-vibrational band of $\mathrm{H}_{3}^{+}$extend right across the planet. Analysis of these features indicates that the jovian disk may be divided into three regions-two mid-high latitude regions with temperatures around $800 \mathrm{~K}$ and a hotter, central region with a temperature around $1200 \mathrm{~K}$. The central meridian longitude of the image in the jovian System III is $102^{\circ}$, coinciding with the region of the Lyman- $\alpha$ Bulge. The significance of new work on the bulge for understanding this image is discussed. The image also shows an interesting doublet centred on $3.52 \mu \mathrm{m}$. Candidates for this feature are discussed; none is found to be satisfactory. 1994 Academic Press, Inc.
\end{abstract}

\section{INTRODUCTION}

Infrared emission due to the $\mathrm{H}_{3}^{+}$molecular ion has now been detected in Jupiter (Drossart et al. 1989), Uranus (Trafton et al. 1993), and Saturn (Geballe et al. 1993). Coupled with significant improvements in the instrumentation now available at infrared observatories, these discoveries have opened up the possibility of using groundbased observations to study ionospheric processes in the jovian planets on a routine basis.

The strong jovian auroral $\mathrm{H}_{3}^{+}$emissions have been the most extensively studied. For example, images taken with new infrared cameras at wavelengths sensitive to $\mathrm{H}_{3}^{+}$ (Baron et al. 1991, Kim et al. 1991) have shown the extent of strong emissions in the auroral regions of Jupiter. These images also show that these emissions vary in intensity on time scales as short as $1 \mathrm{hr}$. Spectroscopic studies of $\mathrm{H}_{3}^{+}$have demonstrated that it plays an important role as a cooling agent in the hot $(T \approx 1000 \mathrm{~K})$ auroral regions of the ionosphere (Joseph et al. 1992) and that both temperature and column density may vary significantly from day to day and according to geographical location. Other spectroscopic studies show that relatively low temperatures may sometimes prevail in the jovian auroral regions (Oka and Geballe 1990).

The addition of significantly more sensitive, long-slit, spectrometers to infrared telescopes means that one can now get simultaneous information about $\mathrm{H}_{3}^{+}$temperatures and column densities and their geographical location on the planet. In this communication we present one such spectral image, obtained from the United Kingdom InfraRed Telescope (UKIRT) located on Mauna Kea, Hawaii. This medium-resolution image is the first to show that $\mathrm{H}_{3}^{+}$emissions extend, at all latitudes, across Jupiter (Miller et al. 1992) and confirms contemporaneous highresolution observations of $\mathrm{H}_{3}^{+}$in the Northern Equitorial Belt, taken with a $5^{\prime \prime}$ aperture on the CanadaFrance-Hawaii telescope (de Bergh et al. 1992).

The detection of a global (dayside) distribution of $\mathrm{H}_{3}^{+}$ 
is not inconsistent with early photochemical models of Jupiter's ionosphere, in which solar EUV ionisation of molecular hydrogen results in $\mathrm{H}_{3}^{+}$formation (e.g., Strobel and Atreya 1983). Indirect evidence of the presence of $\mathrm{H}_{3}^{+}$in the jovian ionosphere had been provided by the in situ measurement by Voyager of magnetospheric $\mathrm{H}_{3}^{+}$ions, presumably of ionospheric origin (Hamilton et al. 1980). The predicted column densities of $\mathrm{H}_{3}^{+}$and its abundance relative to protons also depend on its dissociative recombination rate, the conversion of protons to $\mathrm{H}_{3}^{+}$via vibrationally excited $\mathrm{H}_{2}$ (McElroy 1973, Cravens 1987), additional ionization sources, and the role of vertical plasma drifts in the ionosphere (McConnell et al. 1982). Recent models predict a significant $\mathrm{H}_{3}^{+}$abundance, becoming the main ion (over protons) in the lower levels of the ionosphere above the homopause (e.g., Majeed and McConnell 1991, Kim et al. 1992). (These results must be treated with some caution, however, since they used a rather low rate $-\sim 2 \times 10^{-8} \mathrm{~cm}^{3} \mathrm{sec}^{-1}$-for the rate of $\mathrm{H}_{3}^{+}$dissociative recombination with electrons.) Particularly hot upper atmospheric temperatures have been inferred from Voyager and Pioneer experiments, but the heating mechanism is still undetermined. The range of predicted ionospheric $\mathrm{H}_{3}^{+}$densities and the hot temperatures could produce nonauroral near-IR emission detectable with today's instrumentation.

In addition, Jupiter's upper atmosphere exhibits a particular phenomenon, first reported in 1978 from a rocket experiment and confirmed by the Voyager UVS instrument and IUE (Clarke et al. 1980, 1981), namely the excess of hydrogen Lyman- $\alpha$ emission in the jovian equatorial region and known as the Lyman- $\alpha$ Bulge. This feature has been observed to be fixed around System III longitude $110^{\circ}$, covering the $30^{\circ}$ to $190^{\circ}$ longitude region (Dessler $e t$ al. 1981, Skinner et al. 1988). More recently, an unusually extended bulge has been observed, covering almost the entire equatorial region (McGrath 1991). For a long time, the bulge was thought to be due to a local enhancement in $\mathrm{H}$ abundance increasing the resonant scattering of the solar Lyman $\alpha$. However, high-resolution IUE observations made by Clarke et al. (1991) have shown instead that the jovian line in the bulge region is broadened, thus increasing the scattering of the solar line. The broadening of the jovian line could be caused by local nonthermal processes such as turbulence or thermospheric winds, but the sources of these processes has not been determined (Clarke et al. 1991, ben Jaffel et al. 1993). The $\mathrm{H}_{3}^{+}$spectral image presented in this paper was obtained at the central meridian longitude (CML) of the bulge region and may thus throw a new light on this phenomenon.

\section{OBSERVATIONS}

Infrared observations of Jupiter were made using the cooled grating spectrometer, CGS4, on UKIRT during

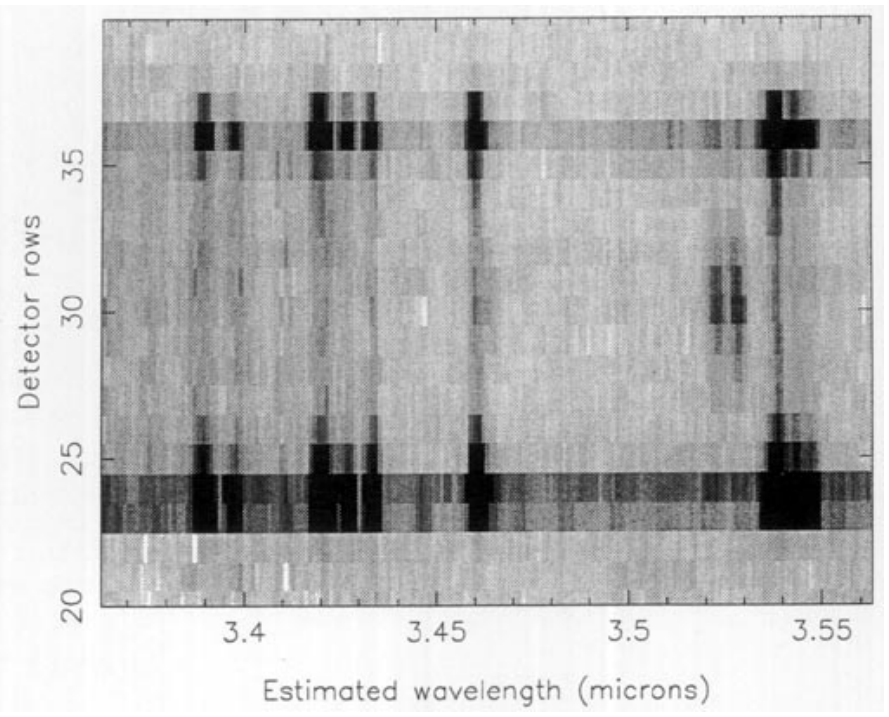

FIG. 1. Spectral image of Jupiter taken on 2 April, 1992, at 10:45 hr UT with a central wavelength of $3.45 \mu \mathrm{m}$ and a resolving power of 1050. North is at the top.

April 1-3 (UT), 1992. Measurements were made using the $150-\mathrm{mm}$ camera and the 150 -lines $/ \mathrm{mm}$ grating in first order, giving a range of resolving powers, $\lambda / \Delta \lambda$, from 1000 to 1300 from 3.2 to $4.1 \mu \mathrm{m}$. With this set up, each pixel in the slit covered an area of 3.1 " square and the wavelength coverage of an individual spectrum was 0.20 $\mu \mathrm{m}$. Using the telescope in chopping mode, spectra were obtained with a total integration time of $4.8 \mathrm{~min}, 2.4 \mathrm{~min}$ on Jupiter and an equal time on the sky.

Seeing on all three nights was better than $1^{\prime \prime}$, as were errors due to telescope pointing and tracking. Total errors could be assessed from a stellar image, using the same integration time. This showed that $95 \%$ of the light from the star was contained within one pixel, indicating that total pointing/tracking/seeing errors were within $\pm 1.5^{\prime \prime}$ (Trafton et al. 1993). The telescope was pointed to align the slit along Jupiter's central meridian longitude, placing the center of the jovian disk on row 30 of the detector array; this pointing was maintained during the 4-min exposure. Since the sub-Earth latitude at the time of the observations was $-1.46^{\circ}$, the jovian equator was also located on row 30 .

The spectral image presented in Fig. 1 is one of a series obtained on April 2, probing jovian emissions between 3.3 and $4.1 \mu \mathrm{m}$. It was taken with the slit aligned north-south along the central meridian. The System III central meridian longitude of the spectrum is $102^{\circ}$, one that has not been associated with highest levels of auroral emission in the L-window in the northern polar regions, although the more diffuse southern aurorae can show strong emission in this region and strong $\mathrm{K}$ window emission due to molecular hydrogen has also been found at this longitude in the 
north. The maximum intensity of the image exceeds 0.55 $\mathrm{pW} \mathrm{m} \mathrm{m}^{-2} \mu \mathrm{m}^{-1}$ in the south and $0.45 \mathrm{pW} \mathrm{m}^{-2} \mu \mathrm{m}^{-1}$ in the north. The scale maximum, however, has been set at 0.05 $\mathrm{pW} \mathrm{m}{ }^{-2} \mu \mathrm{m}^{-1}$ to enhance the low-intensity $\mathrm{H}_{3}^{+}$emission.

Between 3.36 and $3.56 \mu \mathrm{m}$ the spectrum of Jupiter has relatively little continuum emission since incident and diffusely reflected solar radiation at these wavelengths is strongly absorbed by methane lying below the homopause (the $\mathrm{CH}_{4} \nu_{3}$ band is centred on $3.3 \mu \mathrm{m}$ ). The very low continuum level makes it possible to detect $\mathrm{H}_{3}^{+}$emission lines across the entire planet; these lines show up quite strongly in the north and south auroral regions and emit with lower intensities at lower latitudes. $\mathrm{H}_{3}^{+}$gives rise to the intense line emission at $3.533 \mu \mathrm{m}$, together with its shoulder at $3.538 \mu \mathrm{m}$, as well as a series of blends at $3.455,3.413$ (and minor peaks), and $3.384 \mu \mathrm{m}$. Signal-tonoise levels for the spectra may be obtained from Fig. 2. This shows that the $\mathrm{s} / \mathrm{n}$ ratios for the main peaks are at least $30: 1$ in rows 36 and 24 , the brightest rows in the north and south, respectively. In Row 30, typical of the low-intensity $\mathrm{H}_{3}^{+}$emission, $\mathrm{s} / \mathrm{n}$ drops to $5: 1$ for the main peaks.

In addition to the emission features accounted for by $\mathrm{H}_{3}^{+}$, there are a number of other lines visible in the image in Fig. 1. In particular, there is a doublet at 3.517 and $3.522 \mu \mathrm{m}\left(2743\right.$ and $\left.2738 \mathrm{~cm}^{-1}\right)$ that is noteworthy. This doublet shows up most strongly at the equator, decreasing in intensity with increasing latitude. However, its intensity also seems to increase approaching the poles of the planet. The continuum also appears to increase in intensity toward the poles, possibly a result of additional scattering of solar radiation by some form of polar haze and/ or limb effects.

\section{ANALYSIS OF THE $\mathrm{H}_{3}^{+}$FEATURES}

The spectrum from each row of the CGS4 array was ratioed using the B7 star $\alpha$-Leo, which was close to Jupiter during the observation period. In order to obtain column densities and temperatures, features clearly attributable to $\mathrm{H}_{3}^{+}$were fitted using the measured spectral frequencies, calculated Einstein A-coefficients (Kao et al. 1991), and a Gaussian profile with a full-width at half-maximum equivalent to the instrumental resolving power. (The ortho: para ratio, sometimes used as a free parameter in such fits, was fixed at $1: 1$.) The wavelength scale was calibrated from the $\mathrm{H}_{3}^{+}$lines using the laboratory wavelengths of Kao et al. (1991).

The row by row result of this fitting procedure, along with the intensity of the $3.533-\mu \mathrm{m}$ peak is reported in Table I; sample fitted spectra are shown in Fig. 2. As well as the fitted column density, $N\left(\mathrm{H}_{3}^{+}\right)$(assuming thermal equilibrium), Table I shows total $\mathrm{H}_{3}^{+}$column densities, $\hat{N}\left(\mathrm{H}_{3}^{+}\right)$, corrected by multiplying by the cosine of the
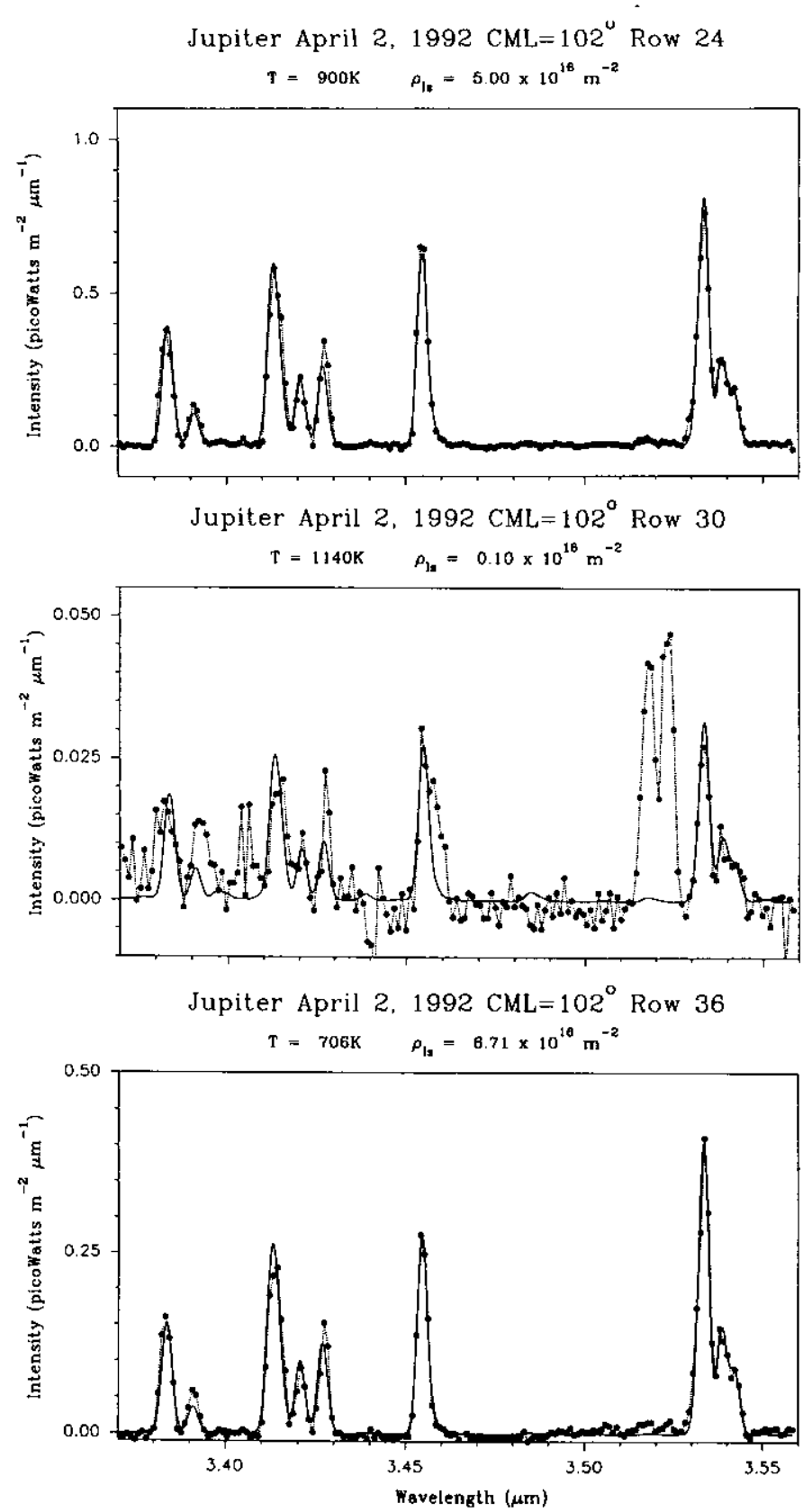

FIG. 2. Sample fitted spectra from individual rows taken from Fig. 1. (Observed spectrum, filled circles; fit, solid line.) As well as the $\mathrm{H}_{3}^{+}$features, the $3.52-\mu \mathrm{m}$ doublet can be seen strongly in row 30 and just above the noise level in the other two rows.

latitude corresponding to the center of the pixel assuming that row 30 was accurately located on the disk center. The table also gives the location of the center of each row on the planet, either in terms the latitude or in terms of kilometers above the limb of the planet, once more assuming that row 30 is correctly centered.

Errors in the temperatures were obtained by fixing the column density to that of the best fit and allowing the 
TABLE I

Fitted $\mathrm{H}_{3}^{+}$Parameters for $3.50-\mu \mathrm{m}$ Spectra on April 2, 1992

\begin{tabular}{ccrrcc}
\hline Row & $\begin{array}{c}I(3.533 \mu \mathrm{m}) \times \\
10^{-16} \mathrm{~W} \mathrm{~m}^{-2}\end{array}$ & $T(\mathrm{~K})$ & $\begin{array}{r}N\left(\mathrm{H}_{3}^{+}\right) \times \\
10^{16} \mathrm{~m}^{-2}\end{array}$ & $\begin{array}{c}\hat{N}\left(\mathrm{H}_{3}^{+}\right) \times \\
10^{16} \mathrm{~m}^{-2}\end{array}$ & $\begin{array}{c}\text { Position } \\
\text { (degrees } / \mathrm{km} .)\end{array}$ \\
\hline 22 & 0.51 & 696 & 0.24 & - & 8000 \\
23 & 18.60 & 680 & 11.54 & 2.31 & 3000 \\
24 & 26.80 & 900 & 5.00 & 1.29 & $\mathrm{~S} 75$ \\
25 & 5.13 & 827 & 1.29 & 0.56 & $\mathrm{~S} 51$ \\
26 & 2.08 & 812 & 0.56 & 0.43 & $\mathrm{~S} \mathrm{39}$ \\
27 & 1.14 & 863 & 0.22 & 0.19 & $\mathrm{~S} \mathrm{28}$ \\
28 & 0.87 & 1230 & 0.07 & 0.07 & $\mathrm{~S} 18$ \\
29 & 1.27 & 829 & 0.28 & 0.28 & $\mathrm{~S} \mathrm{09}$ \\
30 & 1.05 & 1140 & 0.10 & 0.10 & $\mathrm{~S} \mathrm{01}$ \\
31 & 1.10 & 1100 & 0.10 & 0.10 & $\mathrm{~N} \mathrm{06}$ \\
32 & 1.04 & 1250 & 0.08 & 0.08 & $\mathrm{~N} 15$ \\
33 & 1.39 & 1043 & 0.14 & 0.13 & $\mathrm{~N} 25$ \\
34 & 1.58 & 983 & 0.19 & 0.15 & $\mathrm{~N} \mathrm{36}$ \\
35 & 2.84 & 664 & 1.78 & 1.19 & $\mathrm{~N} 48$ \\
36 & 14.49 & 706 & 6.71 & 2.62 & $\mathrm{~N} \mathrm{67}$ \\
37 & 3.82 & 869 & 0.81 & 0.16 & 3000 \\
38 & 0.47 & 819 & 0.13 & - & 8000 \\
\hline
\end{tabular}

Note. $\mathrm{CML}=102^{\circ}$. (See text for discussion of average temperatures and errors.)

temperature to vary to the point that the standard deviatation doubled. This gave errors of $\pm 100 \mathrm{~K}$ for rows with intensities above $2 \times 10^{-16} \mathrm{~W} \mathrm{~m}^{-2}$ and $\pm 200 \mathrm{~K}$ for the less intense rows. Errors in the column density are $\pm 10 \%$ for the brightest rows and $\pm 25 \%$ for the low-intensity region, assuming that the best fit temperature is correct. In the following analysis, we do not consider that rowby-row variations should be treated as significant.

Instead, we divide the data in Table I into three regions-two mid-high-latitude regions of relatively low temperatures $(\sim 800 \mathrm{~K})$ and high column densities and a low-intensity region where column densities are low but temperatures (1000-1250 K) considerably higher, whose center appears to be located to the north of the equator. To improve the signal-to-noise ratio and the statistics of the fits, we have therefore combined the spectra into three groups, depending on the integrated intensity of the 3.533$\mu \mathrm{m}$ peak. In the south we have combined rows 23 to 26 (southern mid-high-latitude region), all of which have the integrated intensity of the $I(3.533)$ peak greater than $2 \times$ $10^{-16} \mathrm{~W} \mathrm{~m}^{-2}$; in the middle of the planet we have taken rows 27 to 34 (central region), all of which have $I(3.533)$ less than this level; and in the north rows 35 to 37 (northern mid-high-latitude region), again with $I(3.533)>2 \times 10^{-16}$ $\mathrm{W} \mathrm{m} \mathrm{m}^{-2}$, have been taken together. These regions correspond to the following latitude ranges: from the south pole to latitude south 33 ; from south 33 to north 42 ; and from north 42 to the north pole.

The fitted temperatures are $813 \pm 41 \mathrm{~K}$ in the south,
$1220 \pm 120 \mathrm{~K}$ in the central region, and $734 \pm 37 \mathrm{~K}$ in the north. Other measurements of the higher latitude regions on April 2, including those coinciding with regions of normally fairly high auroral activity, show that temperatures were generally in the region of $800 \mathrm{~K}$ on both poles, with local maxima of around $900 \mathrm{~K}$ and local minima 700 $\mathrm{K}$. Low intensities of $\mathrm{K}$-band $\mathrm{H}_{3}^{+}$overtone emissions were also recorded during our observations, again indicating a relatively low temperature in the mid-high-latitude regions.

Our observations are the first to show latitudinal variations in the ionospheric temperature independent of local time effects. The temperatures derived in the mid-highlatitude regions are dominated by the intense auroral $\mathrm{H}_{3}^{+}$emission, which probably emanates from altitudes between the $10^{-7}$ - and the $10^{-9}$-bar levels; the emissions in the central region may reflect $\mathrm{H}_{3}^{+}$production at higher-and hotter-ionospheric altitudes due to solar euv radiation.

De Bergh et al. (1992) reported temperatures for their observations in the northern equatorial belt of only 800 $\mathrm{K}$, lower than the auroral temperatures they obtained for the same run, in contrast with our results. The only other nonauroral measurements of the ionospheric temperature come from spacecraft radio occultation data, probing the highest ionospheric levels, where $\mathrm{H}_{3}^{+}$is not dominant ion. These measurements produce temperatures between 600 and $1400 \mathrm{~K}$ (see review by Strobel and Atreya 1983). A more detailed comparison of our data with that from the various spacecraft would require a better knowledge of the altitude distribution of $\mathrm{H}_{3}^{+}$.

The fact that our CML coincides with the Lyman- $\alpha$ Bulge complicates the interpretation of our data. Recent work on the Bulge by ben Jaffel et al. (1992) predicts an upper atmospheric wind system connecting the northern auroral region (latitude $65^{\circ}$, longitude $180^{\circ}$ ) to that of the south (latitude $65^{\circ}$, longitude $0^{\circ}$ ), resulting in elevated temperatures in the equatorial region. The higher temperature we observe in the central region could be a reflection of this process.

Finally, it appears from Table I that there are quite high-intensity $\mathrm{H}_{3}^{+}$emissions coming from off the limb of the planet, as defined by the normal polar radius of Jupiter. Certainly, rows 23 and 37, whose centers we would place about $3000 \mathrm{~km}$ off the limb, have strong emissions; the intensities in rows 22 and 38 are still appreciable, but may be explicable by seeing and other effects. However, further analysis is required to extract the effect of convolving the limb brightening of $\mathrm{H}_{3}^{+}$, the limb darkening of the continuum and the seeing/pointing errors, before the extent of off-limb emission (if any) may be truly determined. This is outside of the scope of the present article and we intend to present a more thorough analysis of this 
phenomenon later, along with the spectra taken at other wavelengths which show similar effects.

\section{THE 3.52- $\mu \mathrm{M}$ DOUBLET}

In addition to the $\mathrm{H}_{3}^{+}$line features, there are two obvious emission lines at 3.517 and $3.522 \mu \mathrm{m}$ whose intensities appear to be almost equal and to maintain a nearly constant ratio with respect to one another across the planet. The intensity of this apparent doublet is greatest at the equator, falling off toward higher latitudes in both the north and the south. Appreciable intensity in the doublet is, however, still apparent at the jovian poles, but no intensity is visible off the planet. From this latter observation we conclude that the doublet is a genuine jovian feature and not simply the result of poor sky subtraction in the images. However, it does not correspond to emission by $\mathrm{H}_{3}^{+}$.

At wavelengths away from the center of its $\nu_{3}$ band, methane is less efficient at absorbing incoming solar radiation and it may be that the doublet marks the onset features due to reflected sunlight. Initial modeling of this effect, however, failed to produce the doublet structure. Another possibility is that collisionally excited ro-vibronic transitions of molecular hydrogen, analogous to electronic transitions of atomic hydrogen such as Pfund- $\gamma(3.740$ $\mu \mathrm{m})$ and $\mathrm{Pf}-\delta(3.296 \mu \mathrm{m})$, are responsible. Unfortunately, the exact wavelengths of the $\mathrm{H}_{2}$ ro-vibronic transitions in our spectral window are not known. The observed doublet also nearly coincides with a quartet of $\mathrm{OH}$ lines; P16.5 1 - at $3.52432 \mu \mathrm{m}, \mathrm{P} 16.51+$ at $3.52234 \mu \mathrm{m}, \mathrm{P} 15.52-$ at $3.52165 \mu \mathrm{m}$, and P15.5 $2+$ at $3.52012 \mu \mathrm{m}$. We have tried to fit these lines to the doublet spectrum obtained by adding the intensities measured in rows 27 to 34 of Fig. 1. The result is far from satisfactory, however. Other likely molecular candidates (neutral or ionic hydrides $\mathrm{C}$, $\mathrm{N}, \mathrm{S}$, or $\mathrm{P}$ ) also fail to fit either the frequencies or the observed splitting.

\section{CONCLUSIONS}

The spectral image presented in this communication shows that $\mathrm{H}_{3}^{+}$emission features extend right across the jovian disk and-at least at CML $102^{\circ}$ - that the derived temperatures are hotter around the equator. Whether the measured increase in ionospheric $\mathrm{H}_{3}^{+}$temperature in the central region is common to all longitudes or particular to the bulge region is currently under investigation. Longterm observations will be required to provide the data necessary to separate the dependence of the jovian ionosphere on solar activity from that due to auroral processes. The spectrum of Jupiter in this part of the Lwindow also contains unidentified features which may later prove useful in probing the dynamics of the jovian upper atmosphere.

\section{ACKNOWLEDGMENTS}

The authors thank Professor Warren Moos for drawing our attention to the possible link between our $\mathrm{H}_{3}^{+}$spectrum and the Lyman- $\alpha$ Bulge. The referees for this paper also made several helpful suggestions. Additionally, we acknowledge Drs. Renée Prange, Lotfi ben Jaffel, John Clarke, Jack McConnell, and Yongha Kim for helpful discussions during the preparation of this communication and thank the staff at UKIRT for their support of these observations.

\section{REFERENCES}

Atreva, S., T. M. Donahue, and J. H. Waite 1979. An interpretation of the Voyager measurement of jovian electron density profiles. $\mathrm{Na}$ ture 280, 795-796.

Baron, R., J. D. Joseph, T. Owen, J. Tennyson, S. Miller, and G. E. BALLESTER 1991. Imaging Jupiter's aurorae from $\mathrm{H}_{3}^{+}$emissions in the 3-4 micron band. Nature 353, 539-542.

ben Jaffel, L., J. T. Clarke, R. Prange, G. R. Gladstone, and A. VIDAL-MaDjar 1993. The Lyman alpha Bulge of Jupiter: Effects of non-thermal velocity field. Geophys. Res. Lett. 20, 747-750.

ben Jaffel, L., J. Sommeria, And R. Prange 1992. A new model for the Lyman alpha Bulge of Jupiter. Bull. Am. Astron. Soc. 24, 1033-1034.

Clarke, J. T., H. A. Weaver, P. D. Feldman, H. W. Moos, W. G. FASTIE, AND C. B. OPAL 1980. Spatial imaging of hydrogen Lyman alpha emission from Jupiter. Astrophys. J. 240, 696-701.

Clarke, J. T., H. W. Moos, and P. D. Feldman 1981. IUE monitoring of the $\mathrm{H}$ Lyman $\alpha$ emission from Jupiter. Astrophys. J. 245, L127.

Clarke, J. T., G. R. Gladstone, and L. Ben Jaffel 1991. Jupiter's dayglow H Ly $\alpha$ emission line profile. Geophys. Res. Lett. 18, 1935-1938.

Cravens, T. E. 1987. Vibrationally excited molecular hydrogen in the upper atmosphere of Jupiter. J. Geophys. Res. 92, 11083-11085.

De Bergh, C., A. Martin, T. Owen, D. Gautier, J.-P. Maillard, B. L. Lutz, AND P. Drossart 1992. Paper presented at July 13-16 Workshop on Variable Phenomena in jovian Planetary Systems held in Annapolis, MD.

Dessler, A., B. R. Sandel, and S. K. Atreya 1981. The jovian hydrogen bulge: Evidence for co-rotating magnetospheric convection. Planet. Space Sci. 29, 215-224.

Drossart, P., J.-P. Maillard, J. Caldwell, S. J. Kim, J. K. G. Watson, W. A. Majewski, J. Tennyson, S. Miller, S. K. Atreya, J. T. Clarke, J. H. Waite, Jr., and R. Wagener 1989. Detection of $\mathrm{H}^{+}$on Jupiter. Nature 340, 539-541.

Geballe, T. R., M.-F. Jagod, and T. OKa 1993. Detection of $\mathrm{H}_{3}^{+}$ infrared emission lines in Saturn. Astrophys. J. Lett., in press.

Hamilton, D. C., G. Gloeker, S. M. Krimigis, C. O. Bostrom, T. P. Armstrong, W. I. AXford, C. Y. FAN, L. J. LANzerotri, AND D. M. HUNTEN 1980. Detection of energetic hydrogen molecules in Jupiter's magnetosphere by Voyager 2: Evidence for an ionospheric plasma source. Geophys. Res. Lett. 7, 813-816.

JosePh, R. D., S. Ridgway, S. Miller, H. A. LAM, AND J. TENNySON 1992. Spectroscopic mapping of $\mathrm{H}_{3}^{+}$emission of Jupiter. Bull. Am. Astron. Soc. 24, 1034.

Kao, L., T. OKa, S. Miller, and J. Tennyson 1991. A table of astronomically important transitions of $\mathrm{H}_{3}^{+}$. Astrophys. J. Suppl. 77, 317-329.

Kim, S. J., P. Drossart, J. Caldwell, J.-P. Maillard, T. Herbst, AND M. SHURE 1991. Images of aurorae on Jupiter from $\mathrm{H}_{3}^{+}$emission at $4 \mu \mathrm{m}$. Nature 353, 536-539. 
Kim, Y. H., J. L. Fox, AND H. S. Porter 1992. On $\mathrm{H}_{3}^{+}$density profiles in the jovian auroral ionosphere. J. Geophys. Res. 97, 6093-7004.

Majeed, T., AND J. C. MCConnell 1991. The upper ionospheres of Jupiter and Saturn. Planet. Space Sci. 39, 1715-1732.

McConnell, J. C., J. B. Holberg, G. R. Smith, B. R. Sandel, D. E. Shemansky, And A. L. Broadfoot 1982. A new look at the ionosphere of Jupiter in the light of UVS occultation results. Planet. Space Sci. 30, 151-170.

McElroy, M. B. 1973. The ionospheres of the major planets. Space Sci. Rev. 14, 460-503.

MCGrath, M. 1991. An unusual change in the jovian Lyman-alpha Bulge. Geophys. Res. Lett. 18, 1931-1934.

Miller, S., T. Geballe, L. M. Trafton, G. E. Ballester, and J. TenNysON 1992. $\mathrm{H}_{3}^{+}$distribution on Jupiter. Bull. Am. Astron. Soc. 24, 1034-1035.
OKA, T., AND T. R. Geballe 1990. Observation of the 4 micron fundamental band of $\mathrm{H}^{+}$in Jupiter. Astrophys. J. 351, L53-L56.

Shemansky, D. E. 1985. An explanation of the H Lyman- $\alpha$ longitudinal asymmetry in the equatorial spectrum of Jupiter: An outcrop of paradoxical energy deposition in the exosphere. J. Geophys. Res. 90, 2673-2692.

Skinner, T. E., M. T. Deland, G. E. Ballester, K. A. Coplin, P. D. Feldman, and H. W. Moos 1988. Temporal variation of the jovian H I Lyman alpha emission. J. Geophys. Res. 93, 29-34.

Strobel, D. F., AND S. Atreya 1983. Ionosphere. In Physics of the Jovian Magnetosphere (A. J. Dessler, Ed.), pp. 51-67. Cambridge Univ. Press, Cambridge.

Trafton, L. M., T. R. Geballe, S. Miller, J. Tennyson, and G. E. BALlester 1993. Detection of $\mathrm{H}_{3}^{+}$from Uranus. Astrophys. J. 405, $761-766$. 